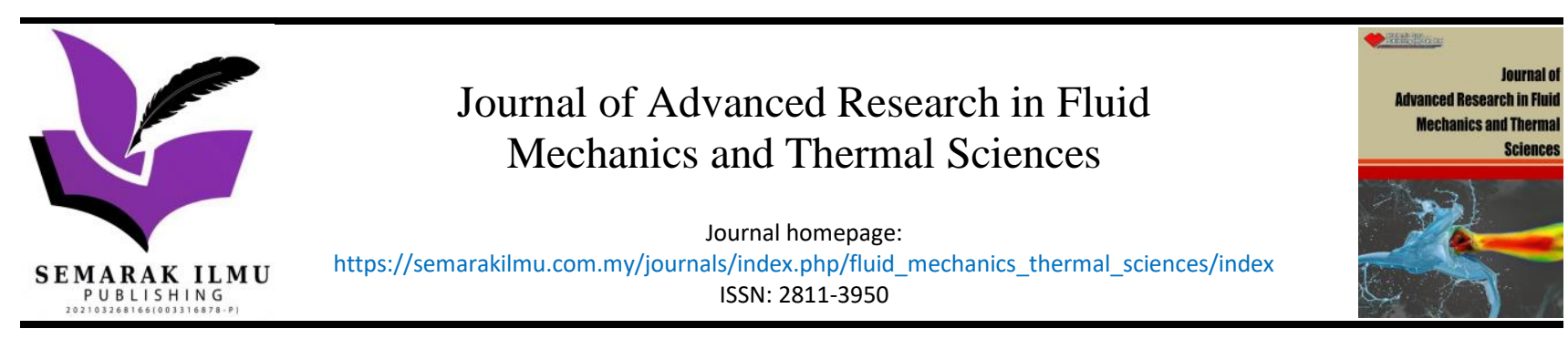

\title{
Flow Reversal of Fully Developed Combined Convection in a Vertical Channel with Boundary Condition of a Third Kind
}

\author{
Nur Asiah Mohd Makhatar ${ }^{1,}{ }^{*}$, Marita Mohd Ali Lee ${ }^{1}$, Nur Lailatulladha Mohamad Fauzi ${ }^{1}$ \\ 1 Department of Mathematics, Faculty of Computer and Mathematical Sciences, Universiti Teknologi MARA, 40450 Shah Alam, Selangor, Malaysia
}

\section{ARTICLE INFO}

\section{Article history:}

Received 19 August 2021

Received in revised form 23 December 2021

Accepted 26 December 2021

Available online 18 January 2022

\section{Keywords:}

Combined Convection; Vertical Channel; Boundary Conditions of Third Kind; Heat Generation

\section{ABSTRACT}

The heat transfer process of combined convection and its flow pattern in a vertical channel is important, especially in environmental, industrial and engineering applications. There has been some concern regarding the heat transfer process in which it is difficult, expensive and time consuming. There are three main objectives to satisfy the purpose of this study which are to determine the impact on flow and heat transfer of the Robin temperature boundary condition, to verify whether the viscous dissipation plays a classical role in fluid flow and heat transfer and lastly to compare the degree of heat transfer facilitated by the boundary conditions of Dirichlet, Neumann and Robin. In this study, the effects of different dimensional parameters were tested and the flow reversal phenomenon was discussed. The Boundary Value Problem (BVP) was solved numerically using Maple using a built-in routine, dsolve. Validation study to the previously published problem was carried out to verify the accuracy of the present computation. The transformation of partial differential equation to ordinary differential equation involved similarity technique. The numerical results of the flow and temperature variables were presented graphically. The flow reversal occurred when the value of internal heat generation (G) and combined convection parameter $(\lambda)$ were large. There was no occurrence of flow reversal when the values of local heating exponent $(\mathrm{p})$ and the Biot Numbers (Bi) increase. However, the value of mass transfer coefficient $(\mathrm{N})$ was different, gave no significant impact.

\section{Introduction}

The theory of heat transfer has been applied mostly in the industrial and engineering field. Heat transfer is the mechanism of the movement of energy with regards to the temperature difference and it is proportional to a temperature gradient. Heat transfer process may involve conduction, convection or radiation. In this present study, convection was applied [3].

Convection is a heat transfer of fluids such as air or water by mass motion as the hot fluid is caused to travel away from the heat source, bringing energy with it. Convection involves the medium between the medium of solid and a fluid but do not involve transfer of heat within a solid. The term convection may be referred to the transfer of mass and or the transfer of heat. The summation of

\footnotetext{
* Corresponding author.

E-mail address: asiah6281@gmail.com
}

https://doi.org/10.37934/arfmts.91.1.5668 
advection and diffusion is called convection, where advection is the heat transported by large-scale movement of currents in the fluid and diffusion is the random Brownian motion of individual particles in the fluid [7].

A lot of studies in heat transfer and fluid flow has been performed in the past few decades involving free, forced or combined convection in a vertical or horizontal channel. A condition where heat is transmitted from both natural and forced convection is known as combined convection. The internal flow may be upwards or downwards on a vertical channel. There were previous researchers who have done researches of combined convection in a vertical heated channel [2], [12], [24]. These studies were then further enhanced by the effect of viscous dissipation and ohmic dissipation by Barletta [4] and Umavathi and Malashetty [42]. The author found out that the effect of viscous dissipation in asymmetrical heating is critical, particularly when it is upward flow because this reduces the effect of flow re-versal. In the case of downward flow, however, it increases this effect. Furthermore, the speed of the fluid rises as the booming forces accelerate because of viscous dissipation. It is also understood that in analytical and numerical solutions, the small value of the perturbation parameter is generally accepted. In addition, [38], investigated fully developed combined viscoelastic fluid convection flow between parallel vertical permeable walls to improve thermal and flow pattern understanding. The combined convection flow characteristics in vertical channels with third form boundary conditions were later analyzed by [44]. The growth of the vortex viscosity parameter has been found to decrease the speed of both aiding and opposing flux. There were a number of researchers who have also examined other problems related to fully developed vertical combined convection flows [5], [6], [8], [11],[14], [16], [20], [21],[23], [26], [29], [30], [33], [39], [46].

Convection is free if the stresses (including the usual pressure) to which the fluid is being exposed at its boundaries do not perform mechanical work. The case where this is not true is termed forced convection referred to as the case where this is not valid. It corresponds to the effect of some mechanical suction pumping on the fluid [25]. Free convection is a kind of movement flow of a liquid such as water or a gas in which no external cause forces the fluid motion. Due to the density difference called free convection, motion within a fluid is created. Some areas are denser than other parts of the fluid. The driving force is a gravity. Since there are hot and cold regions of air or water, free convection will occur, because as they are heated, both water and air become less dense. In addition to increase the heat transfer, forced convection is a particular form of heat transfer in which fluids are forced to move. With a ceiling fan, a pump, suction unit, or another, this forcing can be performed.

A perturbation series method that implements a perturbation parameter proportional to the Brinkman number obtains the velocity field, the temperature field and the Nusselt numbers. Dimensionless coefficients are determined that allow the calculation of the dimensionless assume velocity of the dimensionless bulk temperature and the numbers of Nusselt [4]. Some essential works that have been published on problems of heat transfer in channels by means of combined convection. Due to some engineering applications in the fields of nuclear reactors, heat exchangers, and so forth, the problems have been extended to those with linearly varying wall temperature or constant wall temperature gradient. Many analyses on combined convection with linearly varying wall temperature are focused on cases of fully developed flows. Based on [3] observation, the heattransfer problems of combined convection are expressible in the form of a Helmholtz wave equation in the complex domain. Including the Darcian force, buoyancy force and no-slip wall effect, the nondimensional temperature distribution, heat transfer rate per unit length between two walls, heat carried out by fluid, friction factor, velocity profile and mass flow rate are all analytically derived by Kou and Lu [28]. Barletta [4] expanded the research on laminar and fully developed combined 
convection in a parallel plate vertical channel with prescribed wall with temperatures the viscous dissipation effect. This effect is supposed to be important for fluids with high dynamic viscosity values, as well as for high velocity flows. When viscous dissipation cannot be ignored, the field temperature relies on the velocity field via a nonlinear term in the energy balance equation. The interacting effects of free and forced convection should represent the information for combined convection. It is important to remember that the combined convection heat transfer will vary significantly from its values in both pure free and pure forced convection [43]. Combined convection flows are known as "buoyancy affected flows" [44]. An analytical relationship exists between the decrease in pressure through the porous medium and the viscous and gravitational [10]. The study of fully developed combined convection in a vertical channel continued in [21] by using nanofluid model. On the other research [36] focusing the effect of thermal radiation and viscous dissipation mention that the flow reversal enhanced by the viscous dissipation. [27] studied of the effects of Brownian motion and thermophoresis in the fully developed mixed convection in a vertical channel filled with nanofluid with a heat source or sink.

This study compares three types of boundary conditions, comprising Dirichlet (first kind), Neumann (second kind) and Robin (third kind). In the Dirichlet condition, the value is the set of the unknown function itself while in the Neumann condition, the function's gradient is set in direction normal direction to the boundary. The value sets of the combination of the unknown function and its normal gradient, which is linear in the unknown function is called the Robin condition. [34] and [35] were among the earlier systematic comparisons of the effect of Dirichlet, Neumann and Robin boundary conditions. The Robin boundary condition has been shown to give the most accurate solution. Therefore, in this research, the Robin condition is extended to the default case. It was [13] who called liquids with suspended nanoparticles as nanoliquids. Ever since the development of nanoparticles became a reality, researchers have begun to use nano liquids as a preferred heat transfer medium. Many researchers have done research on mixed convective flows of nanoliquids in channels, including [45]. In this case it was found that as the right nanoliquids are added, the heat transfer characteristic can be considerably improved. [31] showed that the entropy production in the vertical channel is reduced by $\mathrm{TiO}_{2}$-water nanoliquids. Meanwhile [11], [15], [17], [18], [22], [32] [41] and [46] were some similar studies. These works use either the [27] or the [9] single-phase model, and their studies are focused on the temperature state of Dirichlet with mostly no viscous dissipation effect. [30] focused more on the boundary condition of a third kind and the viscous dissipation for the laminar flow in a parallel plate vertical channel with two non-miscible viscous fluid. The latest study found involving boundary condition of a third kind in [1] focused on substantial heat generating or absorbing fluid affected by the viscous dissipation and the thickness of the boundary plate with non-uniform internal temperature. In nanoliquid-based applications, it is now recognized that the situation of 'enhanced heat transfer' is seen only for dilute nanoparticle concentrations. Since this was the case, the nanoliquids have sufficient chance of viscous dissipation and are therefore an important feature to research in vertical channel flows.

Other related studies to the impact of buoyancy on heat transfer was done by [37], where an increase in the fluid temperature is observed when the Grashof number is increased, which was resulted due to the fact that heat transfer due to convection facilitates the flow velocity and therefore the entropy generation. Meanwhile, the study by [40] revealed that the use of correct fluid model is of major importance to understand the behavior and properties of physiological fluids during peristalsis. Later, [19] explored the characteristics of catalytic surface reactions on free convection non-Newtonian nanofluid boundary layers which are driven by buoyancy force. It was revealed that the heat of reaction raises the temperature and concentration profiles significantly due to the catalytic surface reactions and it shows a slight enhancement in the velocity profile. 
The aim of this research is to extend the studies of [4] by taking into consideration that the concentration as well as the temperature walls are constant and on top of that, there is a nonuniform internal heating inside the channel. The investigation is of high importance as it gives an aid to the understanding of flow pattern or thermal characteristics. A simple example on the application on this study is in counter-flow air humidifiers and evaporative cooling of the falling liquid film through interfacial heat and mass transfer in a vertical channel. The basic equations under the flow assumptions are solved numerically after being reduced to ordinary differential equations. Flow and heat transfer results have been reported for various range of values of relevant parameters. The graphical images of the effects of these parameters such like the combined convection parameter, the internal heat generation, the local heating exponent, the concentration parameter, the velocity distribution and the temperature distribution are shown in the results. As far as it is concerned, this problem has not been studied yet.

\section{Methodology}

\subsection{Mathematical Formulation}

In the fully formed parallel vertical channel, we consider the steady flow of a viscous and incompressible fluid. $L$ is the width of the channel, which is the length between the walls. The $x$-axis is selected in such a way that the gravitational acceleration vector $g$ is parallel, but in the opposite way. The $y$-axis is perpendicular to the walls $s$ and the origin of the axes is such that the channel walls are with the positions of $-\frac{L}{2}$ and $\frac{L}{2}$ respectively. The walls at $-\frac{L}{2}$ and $\frac{L}{2}$ are isothermal at given temperatures $T_{C}$ and $T_{H}$, where it is assumed $T_{C}<T_{H}$. The flow has a uniform upward vertical velocity, $U_{0}$ at the channel entrance. The illustration of the geometry and boundary conditions is shown in Figure 1.

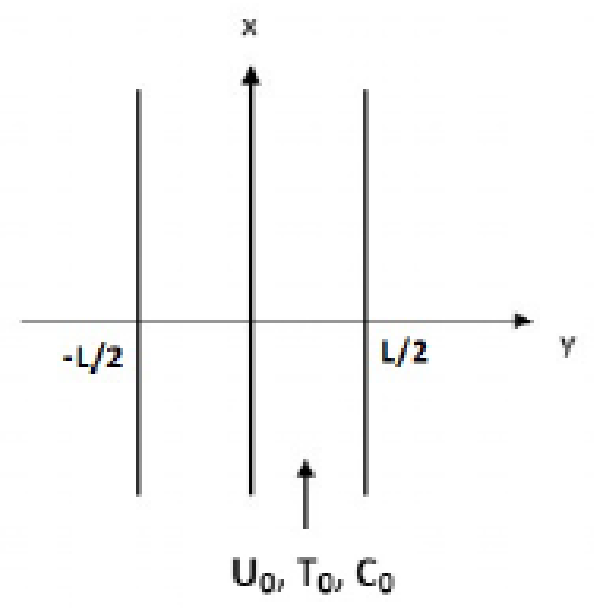

Fig. 1. Physical Configuration

Concentrations are low and the mixture's molecular weights are assumed to be approximately equal. In addition, pressure drops and variations in temperature are low. Thus, with these conditions, it is possible to ignore thermal and pressure diffusion as well as energy diffusion due to the concentration gradient.

The transverse velocity as well as the axial direction temperature and concentration gradients are considered to be zero, so that the flow is a properly developed flow. Therefore, the following equations apply: 


$$
\begin{aligned}
& v=0, \quad \frac{\partial u}{\partial x}=0, \quad \frac{\partial T}{\partial x}=0, \\
& \frac{\partial p}{\partial y}=0, \quad \frac{\partial p}{\partial x}=\frac{d p}{d x}=\text { const }
\end{aligned}
$$

in which $v$ is the velocity component in the ${ }^{y}$-direction and $p$ is the pressure. This will lead us to the governing equations:

$$
\begin{aligned}
& v \frac{\mathrm{d}^{2} u}{\mathrm{~d} y^{2}}+g \beta_{T}\left(T-T_{0}\right)+g \beta_{c}\left(c-c_{0}\right)-\frac{1}{\rho} \frac{\mathrm{d} p}{\mathrm{~d} x}=0, \\
& \alpha \frac{\mathrm{d}^{2} T}{\mathrm{~d} y^{2}}+g\left(T-T_{0}\right)^{p}=0, \\
& \frac{\mathrm{d}^{2} c}{\mathrm{~d} y^{2}}=0 .
\end{aligned}
$$

Where $\rho$ is the density, $v$ is the kinematic viscosity, $c$ is the species mass fraction, $\beta$ is the coefficient of thermal expansion, $T_{0}$ is the reference temperature and it is assumed that $T_{0}$ is given by $T_{0}=\left(T_{C}+T_{H}\right) / 2$.

The governing equations are subjected to the boundary conditions:

$$
u\left(-\frac{L}{2}\right)=u\left(\frac{L}{2}\right)=0 \text {. }
$$

The boundary conditions on the temperature field are assumed to be the following:

$$
\begin{aligned}
& -\left.k_{n l} \frac{\partial T}{\partial Y}\right|_{Y=-\frac{L}{2}}=h_{C}\left[T_{C}-T\left(X,-\frac{L}{2}\right)\right], \\
& -\left.k_{n l} \frac{\partial T}{\partial Y}\right|_{Y=\frac{L}{2}}=h_{H}\left[T\left(X, \frac{L}{2}\right)-T_{H}\right],
\end{aligned}
$$

in which $h_{C}$ and $h_{H}$ are constants.

The reference temperature $\left(T_{0}\right)$ is defined as:

$$
T_{0}=\frac{T_{C}+T_{H}}{2}+S\left(\frac{1}{B i_{C}}-\frac{1}{B i_{H}}\right)\left(T_{H}-T_{C}\right)
$$

Equations (2) to (5) can be written in a dimensionless form by employing these dimensionless parameters: 
$X=\frac{x}{L R e}, \quad Y=\frac{y}{L}, \quad U=\frac{u}{u_{0}}, \quad \operatorname{Re}=\frac{u_{0} L}{v}, \quad \lambda=\frac{G r_{T}}{R e}, \quad \gamma=\frac{\mathrm{d} P}{\mathrm{~d} X}$,

$\theta=\frac{T-T_{0}}{T_{C}-T_{0}}, \quad C=\frac{c-c_{0}}{c_{C}-c_{0}}, \quad P=\frac{p}{\rho u_{0}^{2}}, \quad G=\frac{g L^{2}(\Delta T)^{p-1}}{\alpha}, \quad N=\frac{\beta_{c} \Delta c L^{3}}{\beta_{T} \Delta T}$,

$G r_{T}=\frac{g \beta_{T} \Delta T L^{3}}{v^{2}}, \quad S=\frac{B i_{C} B i_{H}}{B i_{C} B i_{H}+2 B i_{C}+2 B i_{H}}$.

The non-dimensional governing equations and boundary conditions are:

$\frac{\mathrm{d}^{2} U}{\mathrm{~d} Y^{2}}+\lambda(\theta+N C)-\gamma=0$

$\frac{\mathrm{d}^{2} \theta}{\mathrm{d} Y^{2}}+G \theta^{p}=0$

$\frac{\mathrm{d}^{2} C}{\mathrm{~d} Y^{2}}=0$

Subject to the boundary conditions:

$U\left(-\frac{1}{4}\right)=U\left(\frac{1}{4}\right)=0$

$\left.\frac{\mathrm{d} \theta}{\mathrm{d} y}\right|_{y=-\frac{1}{4}}=B i_{C}\left[\theta\left(-\frac{1}{4}\right)+\frac{R_{T} S}{2}\left(1+\frac{4}{B i_{C}}\right)\right]$,

$\left.\frac{\mathrm{d} \theta}{\mathrm{d} y}\right|_{y=\frac{1}{4}}=B i_{H}\left[-\theta\left(\frac{1}{4}\right)+\frac{R_{T} S}{2}\left(1+\frac{4}{B i_{H}}\right)\right]$.

The dimensionless form of velocity and temperature profiles thus depend on five parameters: the combined convection parameter $\left(\lambda=G r_{T} / \operatorname{Re}\right)$, the Biot numbers $\left(\mathrm{Bi}_{1}\right.$ and $\left.\mathrm{Bi}_{2}\right)$, the internal heating parameter $(G)$, mass transfer coefficient $(N)$ and local-heating exponent $(p)$.

\subsection{Solutions Method}

There are various types of analytical or numerical methods which can be employed to solve the nonlinear systems of ordinary differential equations. For this study, though the governing equations seem simple, they are not analytically tractable due to the use of a more realistic Robin boundary condition on temperature. Hence, shooting method is used to solve for the flow and temperature distributions. A build-in routine, dsolve in MAPLE is used to solve the flow and temperature distributions. Few steps were carried out in order to complete this study. The steps consist of transforming partial differential equation to ordinary differential equation manually, solve boundary 
value problem by dsolve from MAPLE, getting the appropriate result and analyzing graph to obtain the conclusion. This summarized in Figure 2.

There seem to be no accurate solutions to the nonlinear boundary value problem (BVP) in system (10)-(12)) when the boundary conditions in Eq. (13)-(15) are applied. Therefore, Maple is used to solve the BVP numerically which is optional in dsolve to address the problem. Maple is a component of math software that combines the world's most sophisticated arithmetic engine with an interface that makes analyzing, exploring, visualizing, and solving mathematical problems. Maple can solve math problems easily and accurately.

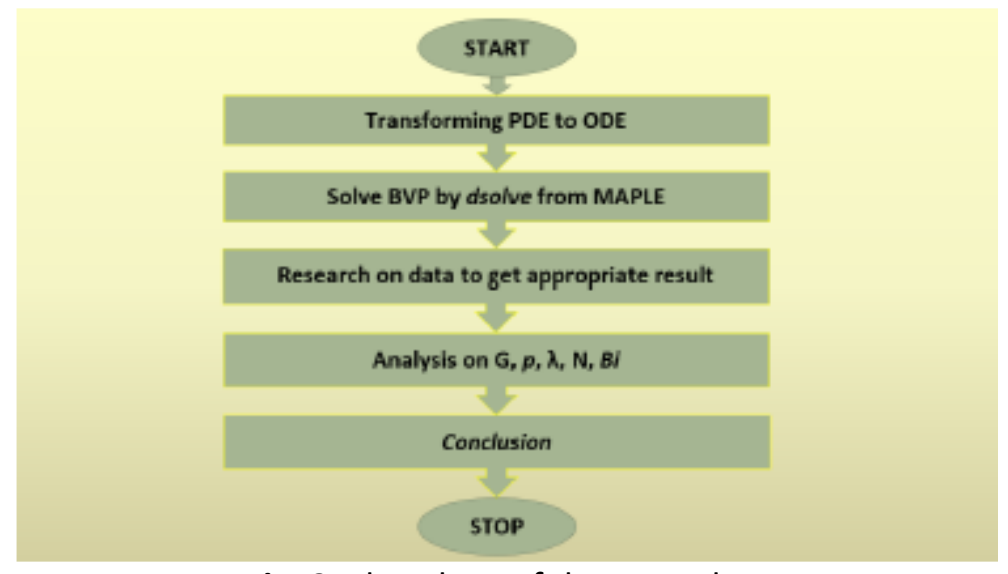

Fig. 2. Flowchart of the procedure

\subsection{Numerical Validation}

In order to validate the numerical code, comparison with a previously published result is necessary. The present numerical results are verified against the result obtained by [4] as shown in Figure 3 for $\mathrm{G}=0$. Clearly, from this comparison, the present results are in excellent agreement with the corresponding result by [4].

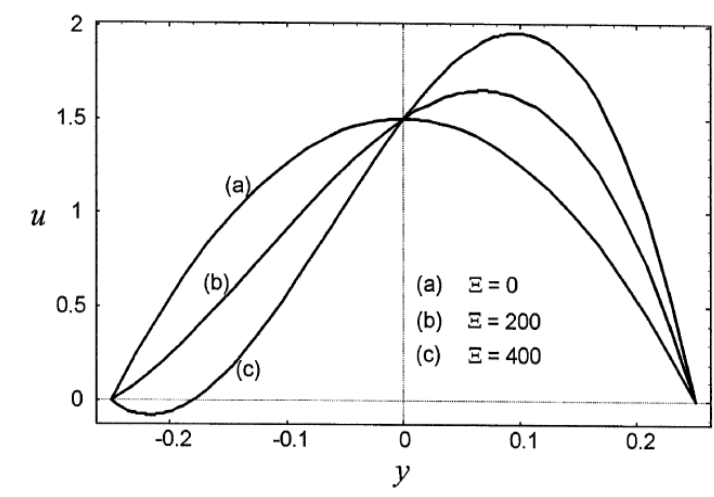

(a)

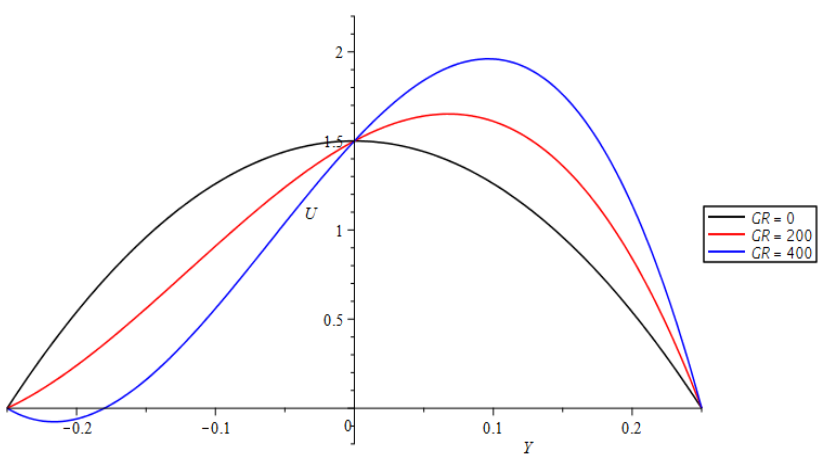

(b)

Fig. 3. Velocity profile for different values of mixed convection parameter (a) Barletta (1998) (b) Present study. 


\section{Results and Discussion}

It is indicated in the foregoing problem that the problem is governed by nondimensional parameters in the following domain: Combined convection parameter $300 \leq \lambda \leq 600$, internal heat generation $10 \leq G \leq 75$, mass transfer coefficient $0.2 \leq N \leq 1$, Biot numbers $0 \leq B i \leq \infty$ and localheating exponent $1.0 \leq p \leq 4.0$. The default values of the parameters are mentioned in the description of the respective figures.

Figure 4 represents the effect of various values of internal heat generation (G) on the velocity profile and temperature profile. It is understood that the negative signs in internal heat generation, $G$ represent heat absorption (cooling), meanwhile positive values of $G$ represent heat generation (heating). Hence, that differentiate between conditions of heat transfer and heat absorption. The focus of this research, however, is on the heat generation paradigm. As the value of $G$ increases, it can be seen in Figure 4(a) that the velocity rises on the right side of the wall and become lower on the left side of the wall. Based on the graph, the flow reversal takes place when $G \geq 50$ and close to cold wall. This is because, the presence of the heat generation results in increasing the region of backflow or the reversal flow zone. On the other hand, the temperature profile of Figure 4 (b) shows that the temperature distribution is linear when $G \leq 10$. When the value of $G$ increases, it can be seen that the temperature rises on the right part of the wall and reduced on the left part of the wall. Increasing $G$ causes the fluid to heat up and therefore increases its temperature to the warmer channel wall from the centre of the channel.

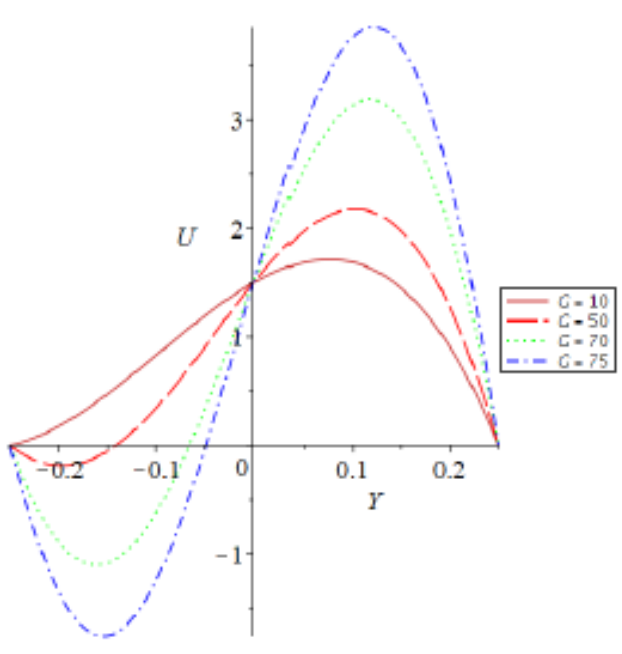

(a)

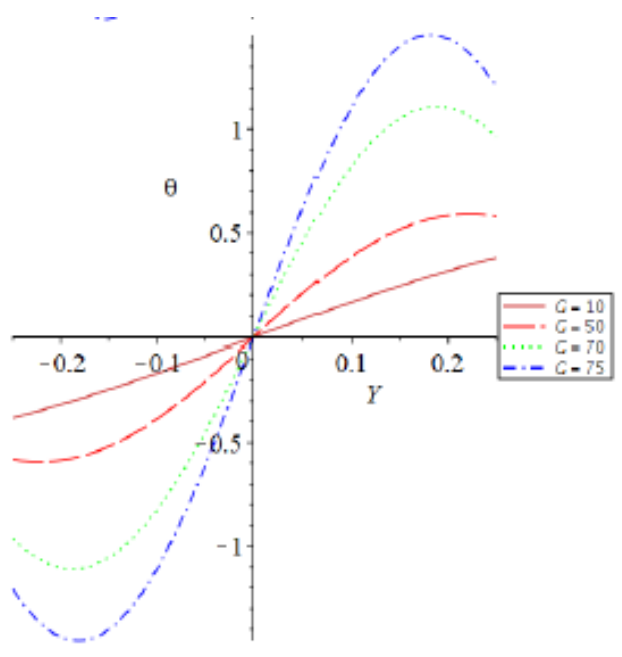

(b)

Fig. 4. (a) Velocity profile and (b) Temperature profile for various values of $G$ where $p=1$, $\mathrm{N}=1, \lambda=300, \mathrm{Br}=2$ and $\mathrm{Bi}_{\mathrm{C}}=\mathrm{Bi}_{\mathrm{H}}=10$

Figure 5 shows the effects of different values of local heating exponent ( $p$ ) on the velocity and temperature profiles. As can be seen in Figure 5(a), the velocity profile increases from the colder wall to the centre of the channel and decrease from the centre to the hotter wall as $p$ increases. The figure also shows that there is no flow reversal occurs and the velocity distributions are asymmetric in relation to the channel's centre and become increasingly so as $p$ grows. Meanwhile, Figure $5(b)$ shows the result of different $p$ values on the temperature profiles. Temperature rises from the colder wall to the channel's centre and decrease afterwards. It can be observed too that as $p$ increases, the results of $p$ on the velocity as well as temperature profile becomes less significant. As $p$ increases, the velocity and temperature profiles reveal no major variation on the flow design. This is because 
the internal heat generation $(G)$ gives more impact on increasing heat generation that it outshined the effects of local heating exponent $(p)$ on heat generation.

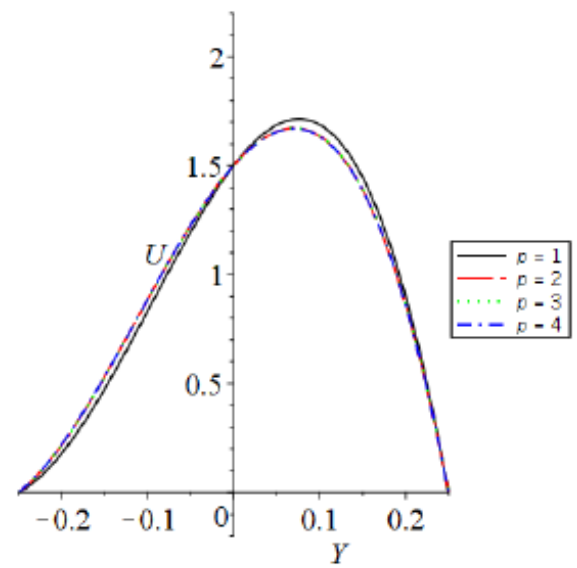

(a)

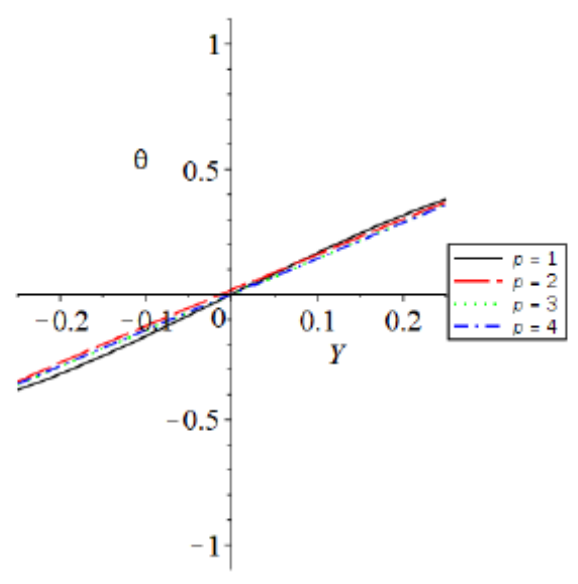

(b)

Fig. 5. (a) Velocity profile and (b) Temperature profile for various values of $p$ where $\mathrm{G}=10, \mathrm{~N}=1, \lambda=300, \mathrm{Br}=2$ and $\mathrm{Bi}_{\mathrm{C}}=\mathrm{Bi}_{\mathrm{H}}=10$

The influence of the combined convection parameter, $\lambda$, on the velocity and temperature profiles can be seen in Figure 6 . With reference to the velocity profile in Figure $6(a)$, the effect of $\lambda$ is more prominent at the cold wall. As the value of $\lambda$ increases, the flow rate decreases, and more flow moves towards the hot channel wall. For large values of $\lambda$, it reaches a point where both pressure and buoyancy mass transfer coefficient lead the fluid adjacent to the cold wall to go backward, resulting in a flow reversal state. There is occurrence of flow reversal in the velocity profile when $\lambda \geq 500$ at the cold wall, meanwhile no flow reversal for $\lambda$ less than 400 . For varied degrees of wall temperature asymmetry, the value of $\lambda$ is required to trigger flow reversal. From Figure $6(\mathrm{~b})$, the changes in the value of $\lambda$ does not affect the temperature design. It also shows that as the value of $\lambda$ increases, the effect of $\lambda$ on the temperature design becomes insignificant.

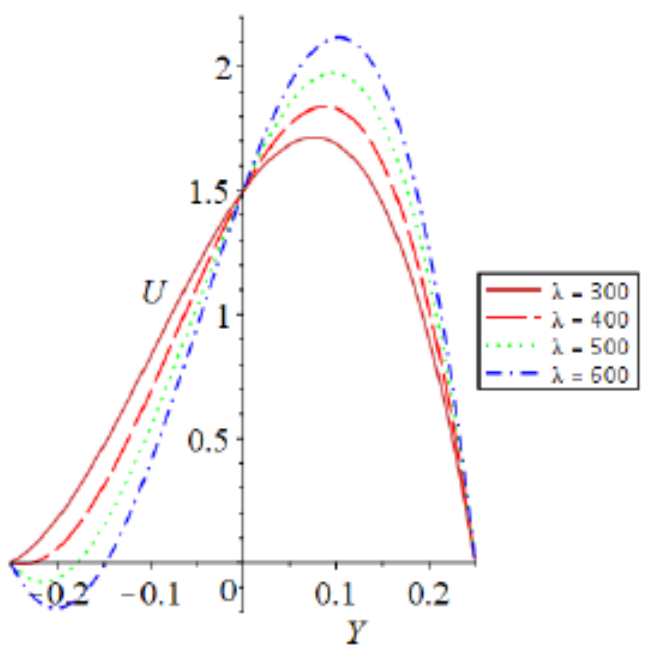

(a)

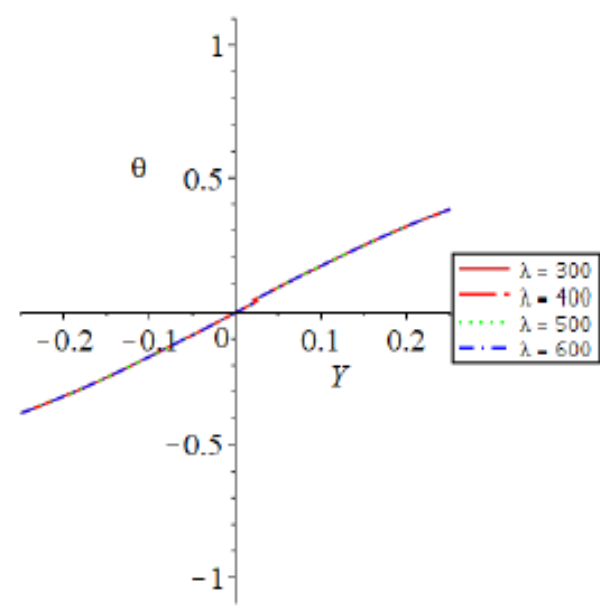

(b)

Fig. 6. (a) Velocity profile and (b) Temperature profile for various values of $\lambda$ where $p=1, N=1$, $\mathrm{G}=10, \mathrm{Br}=2$ and $\mathrm{Bi}_{\mathrm{C}}=\mathrm{Bi}_{\mathrm{H}}=10$ 
Figure 7 shows that as the $\mathrm{N}$ changes, there is no significant changes on the velocity profile and temperature profiles. As the $\mathrm{N}$ increase from -0.2 to 1 , the velocity profile and temperature profile in Figure 7 reveals that no substantial change in the flow pattern occurred. There is no reversal flow that occurs within the boundary condition $Y=-0.25$ to $Y=0.25$. The values are insignificant because the parameter internal heating generation $(G)$ is more dominant compared to mass coefficient transfer (N). In Barletta's research, parameter $\mathrm{N}$ gives an impactful value on heat transfer because there was no parameter $G$ in the study.

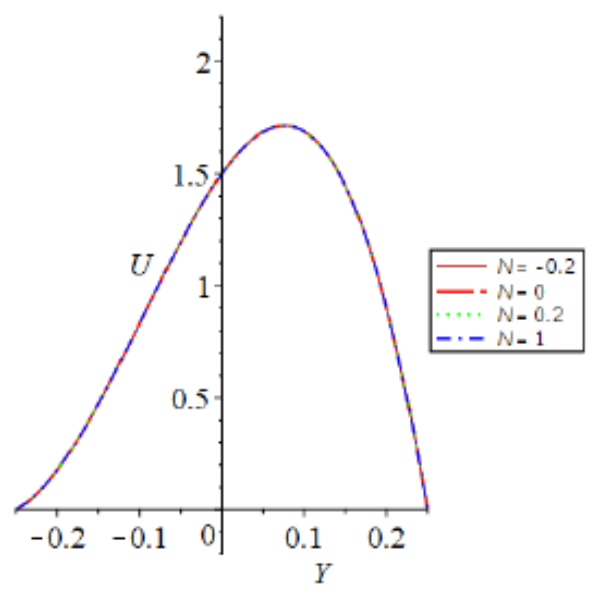

(a)

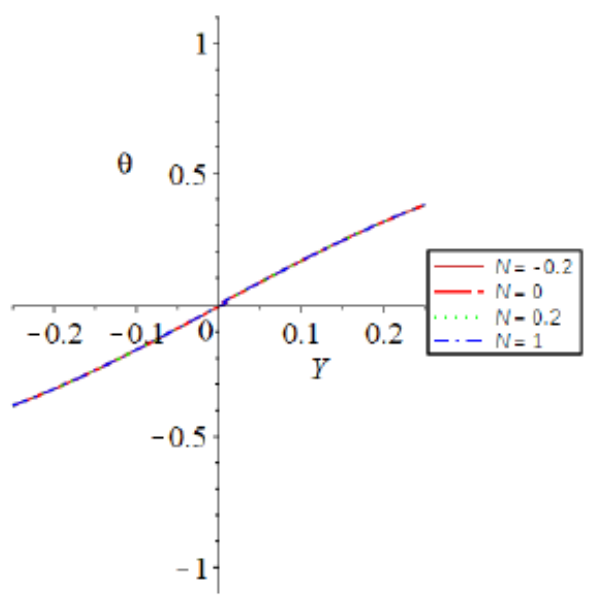

(b)

Fig. 7. (a) Velocity profile and (b) Temperature profile for various values of $\mathrm{N}$ where $\mathrm{p}=1$, $\mathrm{G}=10, \lambda=300, \mathrm{Br}=2$ and $\mathrm{Bi}_{\mathrm{C}}=\mathrm{Bi}_{\mathrm{H}}=10$

Velocity profile and temperature profile for three (3) kinds of Boundary Conditions on temperature i.e $\mathrm{Bi}=0$ is Neumann, $\mathrm{Bi}=10$ is Robin and $\mathrm{Bi} \rightarrow \infty$ is Dirichlet, where $\mathrm{Bi}_{\mathrm{C}}=\mathrm{Bi}_{\mathrm{H}}=\mathrm{Bi}, \lambda=$ $300, p=1$ and $N=1$ is shown in Figure 8. From the graph pattern in Figure 8(a), it can be noticed that the velocity profile decreases with an increase in Biot Number from the cooler wall approaching to the centre of the channel and increase with the increase in Biot number from the centre of the channel approaching the hotter wall. Biot Number $(\mathrm{Bi})$ is the ratio of thermal resistance between the channel and the liquid. For this study, $\mathrm{Bi}_{\mathrm{c}}$ refers to the Biot number on the colder wall (left wall), meanwhile $\mathrm{Bi}_{H}$ refers to the Biot number on the hotter wall (right wall). Higher value of Biot Number (Dirichlet condition) indicates higher magnitude of the velocity as well as temperature profile at the vicinity of the hot wall. From the plot in Figure 8(b), we can say that the temperature profile decreases with the increase in Biot Number, Bi. The changes of $\mathrm{Bi}$ will affect the temperature design which consist of Neumann boundary when the Bi very small, Robin boundary when the $\mathrm{Bi}$ is moderate and Dirichlet when $\mathrm{Bi}$ is approaches infinity. This is because the stronger Buoyancy forces due to the increase in the power of the convective process on the channel resulted to stronger heat transfer. The wall temperature increases with the increase of Biot numbers. It is expected that as Biot number goes to $\infty$, the convective boundary conditions will become the prescribed wall temperature case. We can notice that on the smaller domain of Dirichlet condition, the result would be unrealistic because the region of change reaches the boundary. Meanwhile, the Robin condition is more superior to the Neumann condition. The result obtained support the research done by Novy et al., [23] Bixler [6] where it was concluded that the most accurate condition is the mixed condition (Robin condition), followed by the traction and free surface inclination boundary condition. 


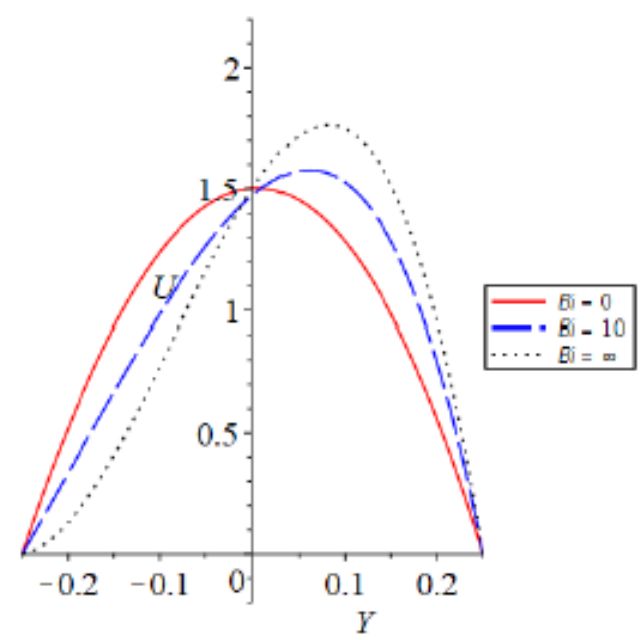

(a)

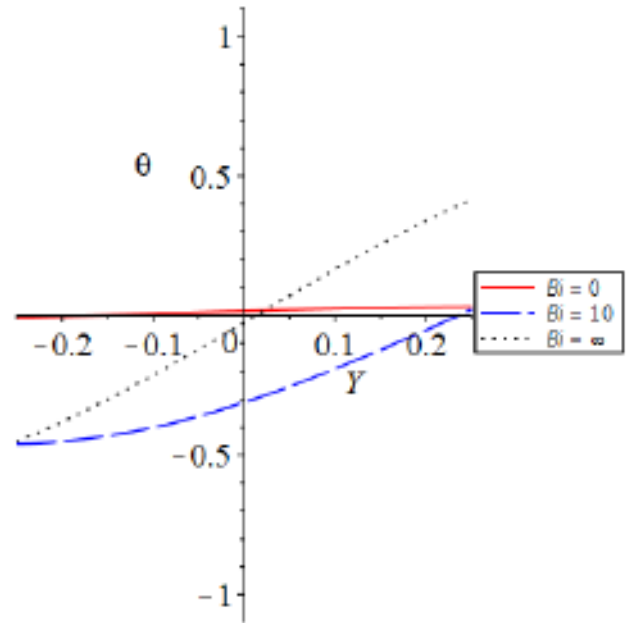

(b)

Fig. 8. (a) Velocity profile and (b) Temperature profile for various values of Biot number: $\mathrm{Bi}=0$ (Neumann), $\mathrm{Bi}=10$ (Robin) and $\mathrm{Bi}=500$ (Dirichlet) where $\mathrm{p}=1, \mathrm{~N}=1, \lambda=300, \mathrm{Br}=2$ and $\mathrm{G}=10$

\section{Conclusions}

In this work, fully-developed mixed convection of heat transfer in a vertical channel by considering Dirichlet, Neumann and Robin boundary conditions has been studied. The dimensionless forms of the governing equations are solved using a built-in routine, dsolve in MAPLE. The major findings are as follows:

i. The liquid cooling is related with a negative value of $G$, meanwhile the positive values correlated to the heat generation case or liquid heating. When there is no internal heat generation ( $G=0$ ) which implies the temperature profile is linear. The temperature profile is asymmetrical because of the asymmetrical nature of the wall temperatures. we can also conclude that the existence of internal heat transfer triggers flow reversal on the cooler wall, which expands the reversal or the return flow area.

ii. Large values of mixed convection parameter $(\lambda)$, enhanced the flow reversal on the velocity profile. The momentum equation is independent of the energy equation in the absence of the combined convection parameter. Therefore, the changes in the value of combined convection $(\lambda)$ does not influence the temperature profile.

iii. The Robin condition appears to be superior to the Neumann condition and the Dirichlet condition seems unrealistic on sufficiently large combined convection parameter $(\lambda)$.

\section{Acknowledgement}

This research was carried out with the support from the Mathematics Department, Faculty of Science Computer and Mathematics, Universiti Teknologi MARA.

\section{References}

[1] Ajibade, A.O. and Umar, A.M. "Steady natural convection couette flow with wall conduction and thermal boundary condition of third kind." ZAMM-Journal of Applied Mathematics and Mechanics/Zeitschrift f"ur Angewandte Mathematik und Mechanik 100, no. 8 (2020): e201900095. https://doi.org/10.1002/zamm.201900095

[2] Aung, W. "Fully developed laminar free convection between vertical plates heated asymmetrically. "International Journal of Heat and Mass Transfer 15, no. 8 (1972): 1577-1580. https://doi.org/10.1016/0017-9310(72)90012-9

[3] Aung, W. and Worku, G. "Theory of fully developed, combined convection including flow reversal. "Journal of Heat Transfer 108, no. 2 (1986): 485-488. https://doi.org/10.1115/1.3246958 
[4] Barletta, A. "Laminar mixed convection with viscous dissipation in a vertical channel. " International Journal of Heat and Mass Transfer 41, no. 22 (1998): 3501-3513. https://doi.org/10.1016/S0017-9310(98)00074-X

[5] Barletta, A., Magyari, E., Pop, I., and Storesletten, L. "Mixed convection with viscous dissipation in a vertical channel filled with a porous medium." Acta Mechanica 194, no. 1 (2007): 123-140. https://doi.org/10.1007/s00707-0070459-3

[6] Barletta, A. and Zanchini, E. "Three-dimensional propagation of hyperbolic thermal waves in a solid bar with rectangular cross-section." International Journal of Heat and Mass Transfer 42, no.2 (1999): $219-229$. https://doi.org/10.1016/S0017-9310(98)00190-2

[7] Bodoia, J. and Osterle, J. "The development of free convection between heated vertical plates." Journal of Heat Transfer 108, no. 2 (1962): 485-488.

[8] Borrelli, A., Giantesio, G., and Patria, M. "Exact solutions in MHD natural convection of a Bingham fluid: fully developed flow in a vertical channel." Journal of Thermal Analysis and Calorimetry, (2021): 1-14. https://doi.org/10.1007/s10973-021-10882-4

[9] Buongiorno, J. "Convective transport in nanofluids." Journal of Heat Transfer 128, no. 3 (2006):240-250. https://doi.org/10.1115/1.2150834

[10] Chamkha, A. J. "Non-Darcy fully developed mixed convection in a porous medium channel with heat generation/absorption and hydromagnetic effects." Numerical Heat Transfer, Part A Applications 32, no. 6 (1997): 653-675. https://doi.org/10.1080/10407789708913911

[11] Chen, B.S. and Liu, C.C. "Heat transfer and entropy generation in fully-developed mixed convection nanofluid flow in vertical channel." International Journal of Heat and Mass Transfer 79, (2014): 750-758. https://doi.org/10.1016/i.ijheatmasstransfer.2014.08.078

[12] Cheng, C.H., Kou, H.S., and Huang, W.H. "Flow reversal and heat transfer of fully developed mixed convection in vertical channels." Journal of Thermophysics and Heat Transfer 4, no. 3 (1970): $375-383$. https://doi.org/10.2514/3.190

[13] Choi, S. U. and Eastman, J. A. "Enhancing thermal conductivity of fluids with nanoparticles." Technical report, Argonne National Lab., IL (United States). (1995): 1-6.

[14] Clift, R., Grace, J. R., and Weber, M. E. "Bubbles, drops, and particles." Courier Corporation. (1978).

[15] Das, S., Jana, R., and Makinde, O. "Mixed convective magnetohydrodynamic flow in a vertical channel filled with nanofluids." Engineering Science and Technology, an International Journal 18, no. 2 (2015): 244-255. https://doi.org/10.1016/i.jestch.2014.12.009

[16] Dubewar, A. and Pandey, H. "Fully developed mixed convection flow of water between two long vertical parallel plates." DAV International Journal of Science 5536, no. 1 (2013): 19-22.

[17] Fakour, M., Vahabzadeh, A., and Ganji, D. "Scrutiny of mixed convection flow of a nanofluid in a vertical channel." Case Studies in Thermal Engineering, no. 4 (2014):15-23. https://doi.org/10.1016/j.csite.2014.05.003

[18] Farooq, U., Hayat, T., Alsaedi, A., and Liao, S. "Heat and mass transfer of two-layer flows of third-grade nano-fluids in a vertical channel." Applied Mathematics and Computation 242, (2014): 528-540. https://doi.org/10.1016/j.amc.2014.05.126

[19] Ganesh, N.V., Kalaivanan, R., Al-Mdallal, Q.M. and Reena, K., "Buoyancy driven second grade nano boundary layers over a catalytic surface with reaction rate, heat of reaction and activation energy at boundary." Case Studies in Thermal Engineering 28: 101346. https://doi.org/10.1016/j.csite.2021.101346

[20] Grosan, T. and Pop, I. "Thermal radiation effect on fully developed mixed convection flow in a vertical channel." Technische Mechanik-European Journal of Engineering Mechanics 27, no. 1 (2007): 37-47.

[21] Grosan, T. and Pop, I. "Fully developed mixed convection in a vertical channel filled by a nanofluid." Journal of Heat Transfer 134, no. 8 (2012): 1-5. https://doi.org/10.1115/1.4006159

[22] Hajipour, M. and Dehkordi, A. M. "Analysis of nanofluid heat transfer in parallel-plate vertical channels partially filled with porous medium." International Journal of Thermal Sciences 55, (2012): $103-113$. https://doi.org/10.1016/j.ijthermalsci.2011.12.018

[23] Hallman, T. M. "Combined forced and free convection in a vertical tube." Purdue University, (1958).

[24] Incropera, F., Kerby, J., Moffatt, D., and Ramadhyani, S. "Convection heat transfer from discrete heat sources in a rectangular channel." International Journal of Heat and Mass Transfer 29, no. 7 (1986): 1051-1058. https://doi.org/10.1016/0017-9310(86)90204-8

[25] Irvine Jr, T., Wu, K., and Schneider, W. J. "Vertical-channel free convection with a power-law fluid." Technical report, Brookhaven National Lab. (1982).

[26] Jha, B. K. and Oni, M. O. "Mixed convection flow in a vertical channel with temperature dependent viscosity and flow reversal: An exact solution." International Journal of Heat Transfer 36, no. 2 (2018): 607-613. https://doi.org/10.18280/ijht.360225 
[27] Khanafer, K., Vafai, K. and Lightstone, M. "Buoyancy-driven heat transfer enhancement in a two-dimensional enclosure utilizing nanofluids." International Journal of Heat and Mass Transfer 46, no. 19 (2003): 3639-3653. https://doi.org/10.1016/S0017-9310(03)00156-X

[28] Kou, H. and Lu, K. "Combined boundary and inertia effects for fully developed mixed convection in a vertical channel embedded in porous media." International Communications in Heat and Mass Transfer 20, no. 3 (1993): $333-345$. https://doi.org/10.1016/0735-1933(93)90019-R

[29] Kumar, J.P., Umavathi, J., Pop, I. and Biradar, B.M. "Fully developed mixed convection flow in a vertical channel containing porous and fluid layer with isothermal or isoflux boundaries." Transport in Porous Media 80, no. 1 (2019): 117-135. https://doi.org/10.1007/s11242-009-9347-8

[30] Kumar, J.P., Umavathi, J.C., Chamkha, A.J. and Ramarao, Y. "Mixed convective heat transfer of immiscible fluids in a vertical channel with boundary conditions of the third kind." Computational Thermal Sciences: An International Journal 9, no. 5 (2017): 447-465. https://doi.org/10.1615/ComputThermalScien.2017019221

[31] Mahian, O., Pop, I., Sahin, A. Z., Oztop, H. F. and Wongwises, S. "Irreversibility analysis of a vertical annulus using Tio2/water nanofluid with MHD flow effects." International Journal of Heat and Mass Transfer 64, (2013): 671679. https://doi.org/10.1016/i.ijheatmasstransfer.2013.05.001

[32] Malvandi, A. and Ganji, D. "Effects of nanoparticle migration on hydromagnetic mixed convection of Alumina/water nanofluid in vertical channels with asymmetric heating." Physica E: Low-dimensional Systems and Nanostructures 66, (2015): 181-196. https://doi.org/10.1016/i.physe.2014.10.023

[33] Michaelides, E. "Particles, bubbles \& drops: their motion, heat and mass transfer." World Scientific, (2006). https://doi.org/10.1142/6018

[34] Novy, R.A., Davis, H.T. and Scriven, L. "A comparison of synthetic boundary conditions for continuous-flow systems." Chemical Engineering Science 46, no. 1 (1991): 57-68. https://doi.org/10.1016/0009-2509(91)80116-G

[35] Papanastasiou, T.C., Malamataris, N. and Ellwood, K. "A new outflow boundary condition." International Journal for Numerical Methods in Fluids 14, no. 5 (1992): 587-608. https://doi.org/10.1002/fld.1650140506

[36] Prasad, K., Mallikarjun, P. and Vaidya, H. "Mixed convective fully developed flow in a vertical channel in the presence of thermal radiation and viscous dissipation." International Journal of Applied Mechanics and Engineering 22, no.1 (2017): 123-144. https://doi.org/10.1515/ijame-2017-0008

[37] Qasim, M., Hayat, K.Z., Khan, I. and Al-Mdallal, Q.M. "Analysis of entropy generation in flow of methanol-based nanofluid in a sinusoidal wavy channel." Entropy 19, no. 10 (2017):490. https://doi.org/10.3390/e19100490

[38] Sajid, M., Pop, I. and Hayat, T. "Fully developed mixed convection flow of a viscoelastic fluid between permeable parallel vertical plates." Computers and Mathematics with Applications 30, (2009): 251-263.

[39] Saleh, H., Hashim, I. and Basriati, S. "Flow reversal of fully developed mixed convection in a vertical channel with chemical reaction." International Journal of Chemical Engineering 2013, (2013): 44-47. https://doi.org/10.1155/2013/310273

[40] Saleem, M., Al-Mdallal, Q.M., Chaudhry, Q.A., Noreen, S. and Haider, A., "Partial slip effects on the peristaltic motion of an upper-convected Maxwell fluid through an irregular channel." SN Applied Sciences 2, no. 5 (2020): 1-14. https://doi.org/10.1007/s42452-020-2457-1

[41] Schlichting, H. "Boundary-Layer Theory." 7th Edition. McGraw-Hill, (1979).

[42] Umavathi, J. and Malashetty, M. "Magnetohydrodynamic mixed convection in a vertical channel." International Journal of Non-Linear Mechanics 40, no. 1 (2005): 91-101. https://doi.org/10.1016/j.ijnonlinmec.2004.05.018

[43] Umavathi, J., Patil, M. B. and Pop, I. "On laminar mixed convection flow in a vertical porous stratum with asymmetric wall heating conditions." International Journal of Transport Phenomena 8, no. 2 (2006): 127.

[44] Umavathi, J. and Veershetty, S. "Non-Darcy mixed convection in a vertical porous channel with boundary conditions of third kind." Transport in Porous Media 95, no. 1 (2012): 111-131. https://doi.org/10.1007/s11242-012-0035-8

[45] Xu, H., Fan, T. and Pop, I. "Analysis of mixed convection flow of a nanofluid in a vertical channel with the Buongiorno mathematical model." International Communications in Heat and Mass Transfer 44, (2013): 15-22. https://doi.org/10.1016/j.icheatmasstransfer.2013.03.015

[46] Xu, H. and Pop, I. "Fully developed mixed convection flow in a vertical channel filled with nanofluids." International communications in heat and mass transfer 39, no. 8 (2012): $1086-1092$. https://doi.org/10.1016/j.icheatmasstransfer.2012.06.003 